\title{
Efeitos da latanoprosta sobre a espessura foveal em olhos submetidos à cirurgia de catarata
}

\author{
Influence of topical latanoprost on foveal thickness in eyes that underwent \\ uneventful cataract surgery
}

\author{
Enyr Saran Arcieri ${ }^{1}$ \\ Camila Naves Mendonça ${ }^{2}$ \\ Daniela Borges Barra ${ }^{3}$ \\ Ticiana Corrêa ${ }^{4}$ \\ Rafael Saran Arcieri ${ }^{5}$
}

\begin{abstract}
RESUMO
Objetivo: Avaliar prospectivamente com o uso da tomografia de coerência óptica se o uso tópico de latanoprosta induz alterações retinianas em pacientes submetidos à cirurgia de catarata. Métodos: Estudo clínico randomizado, com observador mascarado e um mês de duração. Pacientes pseudofácicos foram tratados com latanoprosta $(n=10)$ ou lubrificante ocular uma vez ao dia (grupo controle - placebo) $(n=10)$. Metade dos pacientes de cada grupo possuía capsulotomia posterior (Nd:YAG laser). Avaliamos o status da barreira hemato-retiniana pela medida da espessura retiniana na fóvea com a tomografia de coerência óptica. Exames de tomografia de coerência óptica e medida da acuidade visual foram realizados antes do início do estudo e com 15 e 30 dias de tratamento. Resultados: Não foi observada alteração significante na média da espessura foveal do grupo controle ( $p>0,0610)$. Houve aumento significante na média da espessura foveal nos pacientes tratados com latanoprosta $(p<0,0004)$. Não foi observada alteração na acuidade visual em nenhum paciente. A média da espessura retiniana na fóvea foi significativamente maior no grupo da latanoprosta $(p<0,0007)$. A espessura foveal nos olhos tratados com latanoprosta com cápsula posterior rota foi significativamente maior que a dos pacientes com cápsula íntegra $(\mathrm{p}<0,0461)$. Comparando apenas os pacientes com cápsula posterior íntegra, houve diferença significante da espessura foveal entre os pacientes tratados com latanoprosta $(236,4 \pm 29,4 \mu \mathrm{m})$ e placebo $(197,8 \pm 19,3 \mu \mathrm{m})$ apenas na avaliação realizada com 30 dias de tratamento. Conclusões: Latanoprosta pode levar à quebra da barreira hemato-retiniana em pacientes pseudofácicos. Isso é mais provável de ocorrer em pacientes com cápsula posterior rota.

Número de registro na base de Ensaios Clínicos: ACTRN012606000265572 (www.actr.org.au)

Descritores: Extração de catarata; Prostaglandinas F, sintética/efeitos adversos; Edema macular/induzido quimicamente; Edema macular/diagnóstico; Edema macular /fisiopatologia; Barreira hemato-retiniana/fisiologia; Tomografia de coerencia óptica.
\end{abstract}

\section{INTRODUÇÃO}

Atualmente o uso dos análogos das prostaglandinas (PG), em decorrência do efeito hipotensor e também da comodidade posológica, vem se tornando muito importante no arsenal para o tratamento do glaucoma primário de ângulo aberto, sendo considerado muitas vezes como primeira opção na tentativa de controle da doença ${ }^{(1)}$.

Entretanto, os análogos da $\mathrm{PG}$ também podem estar associados à quebra da barreira hemato-retiniana com o desenvolvimento de edema macular cistóide $(\mathrm{EMC})^{(2-10)}$. 
Embora existam alguns relatos sobre EMC e uveíte anterior associados ao uso de latanoprosta em pacientes pseudofácicos e afácicos ${ }^{(4,10)}$, a incidência desses efeitos adversos é desconhecida.

Existem relatos na literatura demonstrando que o uso de latanoprosta em pacientes com glaucoma e sem história de cirurgia intra-ocular prévia não está associado ao desenvolvimento de $\mathrm{EMC}^{(11)}$. Entretanto, dois relatos de pacientes pseudofácicos ou afácicos que utilizaram análogos da PG no pósoperatório recente ou até mesmo tardio de cirurgia de catarata evidenciaram uma potencial quebra das barreiras hematoaquosa e hemato-retiniana ${ }^{(2-3)}$.

O EMC observado após a cirurgia de catarata foi inicialmente relatado por Irvine em 1953 e é denominado síndrome de Irvine-Gass ${ }^{(12)}$. Aproximadamente $20 \%$ dos pacientes submetidos à cirurgia de catarata (facoemulsificação ou extração extra-capsular) sem intercorrências desenvolvem EMC angiograficamente comprovado, porém redução clinicamente significante da acuidade visual é observada em apenas $1 \%$ desses olhos $^{(13)}$.

Como a cirurgia de catarata isoladamente já pode induzir a alteração da barreira hemato-retiniana com desenvolvimento de EMC e a coexistência de outras doenças, como o glaucoma, pode aumentar esse risco, decidimos avaliar se o uso de latanoprosta em pacientes pseudofácicos sem nenhuma outra doença que pode estar associado ao desenvolvimento de EMC.

Não existe, até o presente momento, nenhum trabalho científico prospectivo, do tipo "mascarado" e randomizado verificando se o uso da latanoprosta está associado ao desenvolvimento de edema macular em pacientes submetidos à cirurgia de catarata utilizando-se a tomografia de coerência óptica (OCT) para avaliar de maneira objetiva a espessura macular. O objetivo primário desse estudo é avaliar prospectivamente, com o uso do OCT, se o uso tópico da latanoprosta em pacientes submetidos à cirurgia de catarata sem intercorrências induz alterações na espessura da região foveal da retina.

\section{MÉTODOS}

Este estudo clínico randomizado, com observador "mascarado" foi realizado no Serviço de Catarata da Universidade Federal de Uberlândia (UFU). O estudo foi conduzido de acordo com os princípios éticos estabelecidos na Declaração de Helsinque após aprovação pelo Comitê de Ética em Pesquisa da UFU. Termo de consentimento livre e esclarecido foi obtido de todos pacientes.

Os pacientes poderiam ser selecionados para participação no estudo desde que os seguintes critérios de inclusão fossem preenchidos: idade maior que 40 anos, presença de cirurgia de catarata com implante de lente intra-ocular sem intercorrências pelo menos há 6 meses, acuidade visual corrigida melhor que 20/40, ausência de doença corneana que dificulte a realização do OCT. Os seguintes critérios de exclusão foram adotados: história pregressa de uveíte ou EMC, irritação ocular importan- te, caracterizada por presença de reação papilar ou hiperemia conjuntival intensa que pudessem ser potencializadas pelo uso da latanoprosta ou do cloreto de benzalcônio, história de outra cirurgia intra-ocular, exceto pela cirurgia de catarata, ou procedimento a laser antes do estudo, indivíduos já tratados com análogos da PG, uso de colírios para tratamento de qualquer doença. Finalmente, a presença de doenças sistêmicas que pudessem estar associadas tanto com uveíte ou EMC (ex: diabetes, doenças reumatológicas), gravidez, lactação ou contracepção inadequada (em mulheres) também foram critérios de exclusão.

Para investigar os efeitos do cloreto de benzalcônio, o conservante utilizado em todos análogos da PG, foi incluído um grupo controle composto por pacientes pseudofácicos que receberam um lubrificante ocular (carboximetilcelulose Lacribell ${ }^{\circledR}$ ), contendo cloreto de benzalcônio, para ser instilado uma vez ao dia. Cada medicamento utilizado no estudo apresenta a seguinte concentração de cloreto de benzalcônio: $0,015 \%$ na latanoprosta e $0,006 \%$ na carboximetilcelulose (placebo).

As medicações avaliadas foram fornecidas em suas embalagens disponíveis no mercado, fabricadas por Pharmacia (latanoprosta - Xalatan ${ }^{\circledR}$ ) e Latinofarma (carboximetilcelulose Lacribell $^{\circledR}$ ).

Para preservar o "mascaramento" foi designado um coordenador não "mascarado" - que não realizou nenhuma avaliação ou medida durante o estudo - para receber os códigos da randomização, entregar as medicações e orientar aos pacientes como utilizar e armazenar os colírios. Os pacientes foram orientados a instilar uma gota de latanoprosta ou placebo uma vez ao dia (20:00 h).

As visitas relativas ao estudo ocorreram no dia do exame inicial e após 15 e 30 dias de tratamento. No início do estudo, os prontuários médicos de cada paciente foram avaliados e os seguintes dados foram obtidos: idade; raça; diagnóstico; status da cápsula posterior; intervalo de tempo entre a cirurgia de catarata e o início do estudo. Em todas as visitas os pacientes foram submetidos à medida da acuidade visual com tabela de Snellen, biomicroscopia, realização de OCT para avaliar a espessura da região foveal e oftalmoscopia. As medidas foram realizadas no mesmo horário durante todas as visitas $(10: 00 \mathrm{~h})$ pelo mesmo observador "mascarado". Para avaliar a espessura foveal com maior acurácia, as imagens de OCT foram repetidas seis vezes e o menor valor foi utilizado como representando a espessura retiniana na fóvea. Para o diagnóstico de EMC utilizamos as imagens fornecidas pelo OCT, considerando a distribuição normal oferecida pelo aparelho abaixo do percentil 5\% $(\mathrm{p}<0,05)$ como indicador de EMC.

O OCT é considerado um método menos invasivo do que a angiofluoresceinografia e possui uma maior sensibilidade para detectar EMC. Alguns autores relataram que o OCT possui uma sensibilidade de $96 \%$ e uma especificidade de $100 \%$ para detectar EMC quando comparado à angiofluoresceinografia ${ }^{(14)}$. 
Os pacientes foram divididos em quatro grupos: grupo A latanoprosta em pacientes com cápsula posterior íntegra, grupo B - latanoprosta em pacientes com cápsula posterior rota, grupo $\mathrm{C}$ - placebo em pacientes com cápsula posterior íntegra, grupo D - placebo em pacientes com cápsula posterior rota.

Antes do estudo, foi determinado que uma amostra de 5 pacientes por grupo de tratamento seria necessária para se ter um poder de $80 \%$ de detectar uma diferença de $20 \%$ na medida da espessura macular entre os grupos empregando-se um nível de significância de 0,05 .

Variáveis categóricas foram analisadas pelo teste exato de Fisher ou teste do Qui-quadrado. Variáveis contínuas foram analisadas pela análise de variância (ANOVA). Alterações dentro dos grupos em relação aos valores iniciais foram analisadas pelo teste $t$ pareado. As diferenças entre os grupos foram declaradas estatisticamente significativas a partir de um valor de $\mathrm{P}=0,05$. Os dados são apresentados como média \pm desvio padrão (DP), exceto onde mencionada outra informação.

\section{RESULTADOS}

Vinte pacientes foram incluídos no estudo: 5 no grupo A, 5 no grupo B, 5 no grupo C e 5 no grupo $\mathrm{D}$. Os dados demográficos e as características clínicas dos grupos de tratamento são apresentados na tabela 1. Não houve diferença estatisticamente significativa entre os grupos em relação ao sexo, idade, raça, média da espessura retiniana na fóvea e intervalo de tempo entre a cirurgia de catarata e o início do estudo.

A tabela 2 apresenta a média da espessura retiniana na fóvea durante todo o estudo. Não houve diferença estatisticamente significante entre os grupos no início do estudo $(\mathrm{p}=0,4929)$. No grupo controle, não foi observado alteração estatisticamente significante na média da espessura foveal durante o acompanhamento ( $>00,0612$ ). Foi observado um aumento estatisticamente significante na média da espessura foveal quando comparados aos valores basais nos pacientes que utilizaram latanoprosta $(\mathrm{p}<0,0032)$.

Nenhum paciente apresentou alteração de sua acuidade visual durante o estudo. Os valores médios da espessura foveal foram significativamente maiores nos pacientes que utilizaram a latanoprosta durante todo acompanhamento. Quando comparando os pacientes tratados com latanoprosta, embora não houvesse diferença estatisticamente significante entre aqueles com cápsula posterior íntegra e os com cápsula rota no início do estudo, houve um aumento significativamente maior na espessura foveal nos pacientes com cápsula rota $(\mathrm{p}<0,0461)$ durante todo o acompanhamento.

Comparando apenas os pacientes com cápsula posterior íntegra, houve uma diferença estatisticamente significante da espessura foveal entre os pacientes tratados com latanoprosta $(236,4 \pm 29,4 \mu \mathrm{m})$ e placebo $(197,8 \pm 19,3 \mu \mathrm{m})$ apenas na avaliação realizada com 30 dias de tratamento $(p=0,0399)$. Entre os pacientes com cápsula posterior rota, os valores da média da espessura foveal foram significativamente maiores nos pacientes tratados com latanoprosta quando comparados ao grupo controle durante todo o acompanhamento (15 dias e 30 dias) $(\mathrm{p}<0,0001)$.

A média das alterações na espessura foveal após utilização de latanoprosta ou placebo estão listadas na tabela 3. Durante o estudo, três pacientes tratados com latanoprosta apresentaram EMC ao OCT considerando a distribuição normal oferecida pelo aparelho $(\mathrm{p}<0,05)$, sendo um com cápsula posterior íntegra e dois com cápsula rota. Como nenhum desses pacientes queixou de alteração de sua acuidade visual ou metamorfopsia, acreditamos que o EMC apresentado tenha sido subclínico. Nenhum paciente

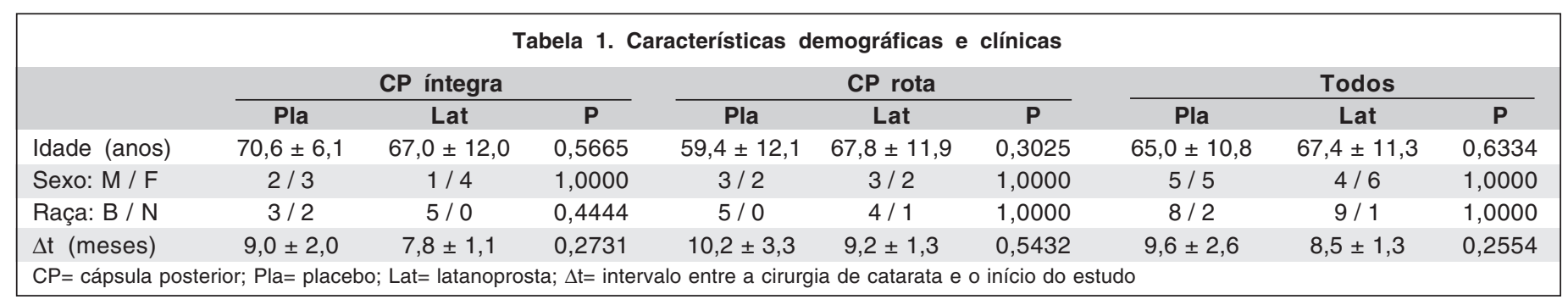

\begin{tabular}{|c|c|c|c|c|c|c|c|c|c|}
\hline & \multicolumn{3}{|c|}{ CP íntegra } & \multicolumn{3}{|c|}{ CP rota } & \multicolumn{3}{|c|}{ Todos } \\
\hline & Pla & Lat & $\mathbf{P}$ & Pla & Lat & $\mathbf{P}$ & Pla & Lat & $\mathbf{P}$ \\
\hline Início & $194,6 \pm 19,9$ & $196,0 \pm 26,1$ & 0,9264 & $194,6 \pm 13,3$ & $181,8 \pm 10,5$ & 0,1302 & $194,6 \pm 15,9$ & $188,9 \pm 20,2$ & 0,4929 \\
\hline 15 dias & $197,0 \pm 16,0$ & $227,2 \pm 39,1$ & 0.1487 & $200,0 \pm 16,9$ & $271,2 \pm 14,6$ & $0,0001^{*}$ & $198,5 \pm 15,6$ & $249,2 \pm 36,2$ & $0,0082^{*}$ \\
\hline$P$ & 0,3812 & $0,0312^{* *}$ & & 0,1265 & $<0,0001^{* *}$ & & 0,0613 & $<0,0001^{\star *}$ & \\
\hline 30 dias & $197,8 \pm 19,3$ & $236,4 \pm 29,4$ & $0.0399^{*}$ & $195,8 \pm 18,0$ & $277,8 \pm 16,8$ & $0,0001^{*}$ & $196,8 \pm 17,7$ & $257,1 \pm 31,4$ & $<0,0001^{*}$ \\
\hline$P$ & 0,0785 & $0,0165^{* *}$ & & 0,6995 & $<0,0001^{* *}$ & & 0,1875 & $<0,0001^{* *}$ & \\
\hline
\end{tabular}


do grupo controle apresentou EMC (Tabela 4). As figuras 1 e 2 ilustram casos de dois pacientes, um com cápsula íntegra e outro com cápsula rota, que desenvolveram EMC. Todos os casos de EMC resolveram após interrupção do uso da latanoprosta.

\section{DISCUSS ÃO}

Embora existam alguns relatos de EMC e uveíte anterior associados ao uso de latanoprosta em pacientes pseudofácicos e afácicos ${ }^{(3-8)}$, a incidência desses efeitos adversos é desconhecida. Vários relatos isolados têm descrito, retrospectivamente, o desenvolvimento de EMC em pacientes pseudofácicos ou afácicos utilizando análogos da PG. ${ }^{(3-10)} \mathrm{Na}$ literatura existem, entre relatos de casos, estudos retrospectivos, estudos de revisão da literatura e estudos prospectivos, aproximadamente 160 casos de EMC possivelmente associados ao uso de análogos da PG, sendo a maioria destes relacionados ao uso de latanoprosta, provavelmente pelo fato desta medicação estar a mais tempo disponível no mercado.

Outros autores analisaram relatos de casos de EMC associados ao uso de latanoprosta, totalizando 100 olhos de 90 pacientes, descrevendo as seguintes condições possivelmente associadas ao risco de desenvolvimento de EMC: cirurgia de catarata; facectomia intra-capsular; afacia; implante de lente intra-ocular (LIO) na câmara anterior; cápsula posterior rota (secundário à cirurgia ou à capsulotomia com Nd:YAG laser); vitrectomia anterior; cirurgia de retina ou vítreo-retiniana; história de uveíte; uveíte concomitante; fibrose pré-macular; história prévia de $\mathrm{EMC}$; retinopatia diabética; oclusão venosa retiniana; pressão intra-ocular (PIO) tratada $<10 \mathrm{mmHg}$; cirurgia filtrante ou outra cirurgia intra-ocular (exceto cirurgia de catarata) no período de até um ano antes do EMC; tratamento concomitante com epinefrina; ceratoplastia penetrante; e doença retiniana inflamatória ${ }^{(15)}$.

Em um estudo foram avaliados 68 olhos de 38 pacientes com glaucoma e sem história de cirurgia intra-ocular prévia que utilizaram latanoprosta durante 6 meses e foram acompanhados com a realização de OCT antes do início do estudo e após 1, 3 e 6 meses do uso de latanoprosta. Nesse estudo o uso de latanoprosta não interferiu na espessura retiniana na região foveal, não estando associado ao desenvolvimento de $\mathrm{EMC}^{(11)}$.

Outros autores analisaram retrospectivamente 162 olhos de 145 pacientes consecutivos submetidos à facoemulsificação

\begin{tabular}{|c|c|c|c|c|c|c|c|c|c|}
\hline & \multicolumn{3}{|c|}{ CP íntegra } & \multicolumn{3}{|c|}{ CP rota } & \multicolumn{3}{|c|}{ Todos } \\
\hline & Pla & Lat & $\mathbf{P}$ & $\mathrm{Pla}$ & Lat & $\mathbf{P}$ & $\mathrm{Pla}$ & Lat & $\mathbf{P}$ \\
\hline 15 dias & $2,4 \pm 5,4$ & $31,2 \pm 21,4$ & $0,0163^{*}$ & $5,4 \pm 6,3$ & $89,4 \pm 13,4$ & $<0,0001^{*}$ & $3,9 \pm 5,8$ & $60,3 \pm 35,0$ & $0,0004^{*}$ \\
\hline 30 dias & $3,2 \pm 3,0$ & $40,4 \pm 22,5$ & $0,0090^{*}$ & $1,2 \pm 6,4$ & $96,0 \pm 17,2$ & $<0,0001^{*}$ & $2,2 \pm 4,9$ & $68,2 \pm 34,8$ & $0,0002^{*}$ \\
\hline
\end{tabular}

\begin{tabular}{|c|c|c|c|c|c|c|}
\hline \multirow[b]{2}{*}{ EMC } & \multicolumn{2}{|c|}{ CP íntegra } & \multicolumn{2}{|c|}{ CP rota } & \multicolumn{2}{|c|}{ Todos } \\
\hline & Pla & Lat & Pla & Lat & Pla & Lat \\
\hline Sim & 0 & 1 & 0 & 2 & 0 & 3 \\
\hline Não & 5 & 4 & 5 & 3 & 10 & 7 \\
\hline $\mathrm{P}$ & 1,0000 & & 0,4444 & & 0,2105 & \\
\hline
\end{tabular}
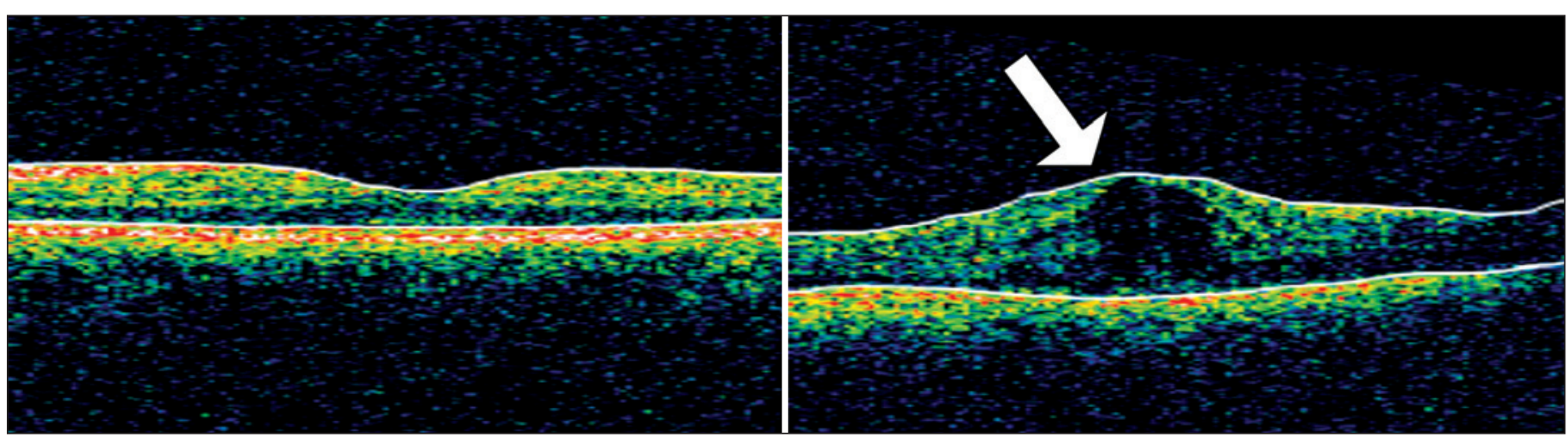

Figura 1 - OCT de um paciente com cápsula posterior rota (Nd:YAG laser) antes do tratamento com latanoprosta (esquerda) e após 30 dias de tratamento com latanoprosta (direita). A imagem da direita mostra a presença de edema macular cistóide (seta) após o uso de latanoprosta. 


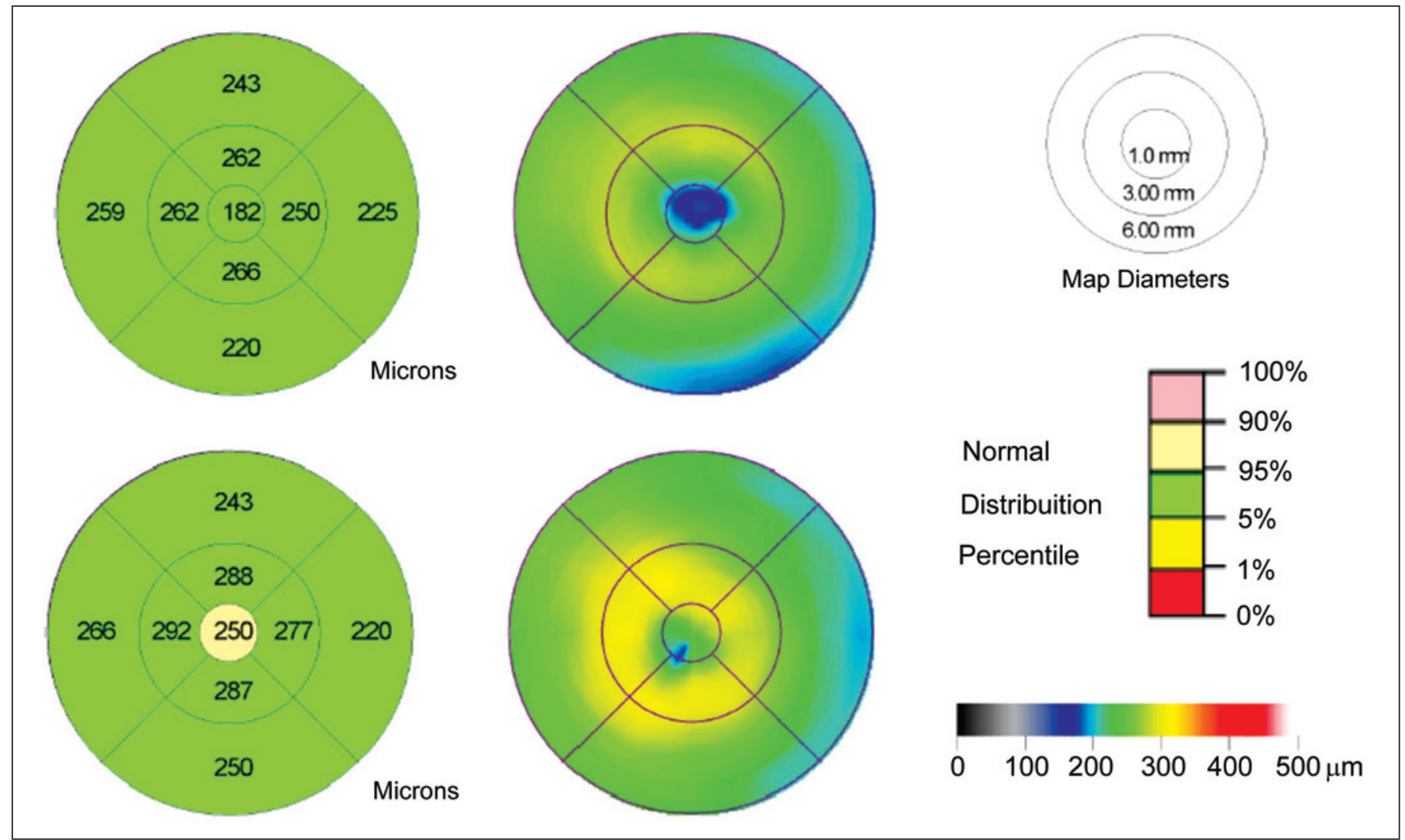

Figura 2 - Medida da espessura macular (em $\mu \mathrm{m}$ ) e mapa de cores de um paciente com cápsula posterior íntegra antes (superior) e após (inferior) 30 dias de uso de latanoprosta. Observou-se um aumento na espessura foveal comparado ao exame inicial.

para determinar a possível associação entre o uso pré-operatório de latanoprosta e o desenvolvimento de EMC no pósoperatório $^{(16)}$. Dos pacientes que vinham usando latanoprosta, oito foram orientados a suspender o seu uso uma semana antes da cirurgia e nenhum desenvolveu EMC. Dos quatro pacientes que não interromperam o uso da latanoprosta, todos apresentaram EMC no pós-operatório ( $\mathrm{p}=0,003)$. Nenhum dos pacientes que desenvolveram EMC um mês após a cirurgia de catarata tinha diabetes mellitus ou qualquer doença sistêmica ou moléstia inflamatória ocular. Esses pacientes apresentaram piora da acuidade visual, porém nas visitas subseqüentes todos os casos de EMC evoluíram para resolução do edema e recuperação da acuidade visual com o término do uso do análogo da PG e tratamento com cetorolaco de trometamina.

A maioria dos casos relatados de EMC associado ao uso de latanoprosta ${ }^{(4-8)}$ ocorreu em pacientes que apresentavam condições oculares e/ou sistêmicas coexistentes que aumentavam o risco de desenvolvimento de EMC, como história prévia de EMC ou uveíte anterior, presença de membrana epiretiniana, oclusão venosa, cirurgia de catarata com complicações, cápsula posterior rota ou ausente, presença de LIO de câmara anterior ou diabetes mellitus.

Embora a patogênese seja provavelmente multifatorial, alguns investigadores acreditam que a inflamação, incluindo mediadores químicos endógenos como as PGs, seja o principal fator etiológico no desenvolvimento de EMC após cirurgia de catarata $^{(3,16)}$.

A ocorrência de inflamação ocular em pacientes submetidos à cirurgia de catarata, com a síntese de PGs, resulta na quebra das zônulas de adesão dos capilares retinianos perifoveais, o que pode estar associado ao desenvolvimento de EMC nesses pacientes. A inibição do EMC do afácico por meio do uso profilático de drogas antiinflamatórias não hormonais fornece suporte a esta teoria ${ }^{(17)}$.

$\mathrm{O}$ fato de nenhum paciente tratado com placebo ter apresentado aumento significativo da espessura foveal sugere que o cloreto de benzalcônio não deve ser o responsável pelas alterações observadas no estudo.

Devemos escolher um plano de tratamento adequado com a melhor relação risco/benefício para nossos pacientes ${ }^{(9)}$. Esse estudo sugere que o uso de latanoprosta pode levar a uma quebra da barreira hemato-retiniana e o desenvolvimento de EMC em olhos submetidos à cirurgia de catarata, mesmo na ausência de intercorrências durante a cirurgia, história pregressa de uveíte ou EMC. Recomenda-se cautela quando da prescrição de latanoprosta para pacientes pseudofácicos, principalmente naqueles com cápsula posterior rota. Se houver necessidade de iniciar o uso de algum análogo da PG em um 
paciente com essas características, torna-se necessário um monitoramento cuidadoso da BHR (com exame à lâmpada de fenda, biomicroscopia de mácula, avaliação da acuidade visual e, se possível, realização de OCT). Se houver necessidade de iniciar o uso de algum análogo da PG em um paciente com essas características, torna-se necessário um monitoramento cuidadoso da BHR (com exame à lâmpada de fenda, biomicroscopia de mácula, avaliação da acuidade visual e, se possível, realização de OCT). Não acreditamos, no entanto, que o uso de análogos da PG nesses casos constitui-se em contra-indicação absoluta, uma vez que a interrupção do tratamento e instituição de colírios antiinflamatórios resulta em resolução do EMC.

\section{CONCLUSÃO}

$\mathrm{O}$ aumento da espessura foveal, medido por meio do OCT, nos pacientes submetidos à cirurgia de catarata em uso de latanoprosta por 30 dias foi significativamente maior no grupo de pacientes com cápsula posterior rota quando comparado aos pacientes com cápsula íntegra, sugerindo uma maior cautela quando utilizar esse tipo de medicamento em pacientes que apresentem cápsula rota. Não existe um único medicamento ou tratamento cirúrgico sem efeitos colaterais ou possibilidade de complicações. Dessa forma, é fundamental estar consciente dos potenciais riscos de qualquer terapia na escolha do tipo de tratamento a ser utilizado.

\section{ABSTRACT}

Purpose: To study prospectively using optical coherence tomography whether topical latanoprost induces retinal disorders in patients that underwent cataract surgery. Methods: Randomized, masked-observer, one-month clinical trial. Pseudophakic patients were treated with latanoprost $(n=10)$ or lubricant drop q.d. (control group) ( $\mathrm{n}=10)$. Half of the patients of each group presented ruptured posterior capsule $(\mathrm{Nd}: \mathrm{YAG}$ laser). We evaluated the blood-retinal barrier status assessed by optical coherence tomography measurement of retinal thickness in the fovea. Before the beginning of the study and after 15 and 30 days of treatment, optical coherence tomography images were taken, and the visual acuity examination was performed. Results: There was no statistically significant increase in mean foveal thickness when patients instilled placebo $(\mathrm{P}>0.0610)$. A statistically significant increase in retinal thickness in the fovea was observed when patients instilled latanoprost $(\mathrm{P}<0.0004)$. No changes were observed in visual acuity in both groups. Mean retinal thickness in the fovea was significantly higher in the latanoprost group $(\mathrm{P}<0.0007)$. The mean foveal thickness in latanoprost treated eyes with ruptured posterior capsule was statistically greater when compared with that of intact posterior capsule $(\mathrm{P}<0.0461)$. When comparing only the patients with that of intact posterior capsule, there was a statistically significant difference in foveal thickness between patients treated with latanoprost $(236.4 \pm 29.4 \mathrm{~mm})$ and placebo $(197.8 \pm 19.3 \mathrm{~mm})$ only at 30 days of treatment. Conclusions: Latanoprost may lead to disruption of the blood-retinal barrier in pseudophakic patients, and is more probable to occur in patients with ruptured posterior capsule.

\section{ACTRN012606000265572 (www.actr.org.au)}

Keywords: Cataract extraction; Prostaglandins F, synthetic/ adverse effects; Macular edema/chemically induced; Macular edema/diagnosis; Macular edema/physiopathology; Bloodretinal barrier/physiology; Tomography, optical coherence

\section{REFERÊNCIAS}

1. Alexander CL, Miller SJ, Abel SR. Prostaglandin analog treatment of glaucoma and ocular hypertension. Ann Pharmacother. 2002;36(3):504-11.

2. Arcieri ES, Santana A, Rocha FN, Guapo GL, Costa VP. Blood-aqueous barrier changes after the use of prostaglandin analogues in patients with pseudophakia and aphakia: a 6-month randomized trial. Arch Ophthalmol. 2005;123(2):186-92.

3. Miyake K, Ota I, Maekubo K, Ichihashi S, Miyake S. Latanoprost accelerates disruption of the blood-aqueous barrier and the incidence of angiographic cystoid macular edema in early postoperative pseudophakias. Arch Ophthalmol. 1999;117(1):34-40. Comment in: Arch Ophthalmol. 1999;117(9):1265-6.

4. Lima MC, Paranhos A Jr, Salim S, Honkanen R, Devgan L, Wand M, et al. Visually significant cystoid macular edema in pseudophakic and aphakic patients with glaucoma receiving latanoprost. J Glaucoma. 2000;9(4):317-21.

5. Warwar RE, Bullock JD, Ballal D. Cystoid macular edema and anterior uveitis associated with latanoprost use. Experience and incidence in a retrospective review of 94 patients. Ophthalmology. 1998;105(2):263-8. Comment in: Ophthalmology. 1998;105(11):1978-81; Ophthalmology. 1998;105(11):1978; author reply 1980-1; Ophthalmology. 1998;105(11):1981-3.

6. Ayyala RS, Cruz DA, Margo CE, Harman LE, Pautler SE, Misch DM, et al. Cystoid macular edema associated with latanoprost in aphakic and pseudophakic eyes. Am J Ophthalmol. 1998;126(4):602-4.

7. Moroi SE, Gottfredsdottir MS, Schteingart MT, Elner SG, Lee CM, Schertzer $\mathrm{RM}$, et al. Cystoid macular edema associated with latanoprost therapy in a case series of patients with glaucoma and ocular hypertension. Ophthalmology. 1999; 106(5):1024-9

8. Watanabe K, Hayasaka S, Hayasaka Y, Nagaki Y, Watanabe K. Cystoid macular edema associated with latanoprost use in a pseudophakic eye with a history of surgical complications. Jpn J Ophthalmol. 2003;47(1):110-2.

9. Wand M, Shields BM. Cystoid macular edema in the era of ocular hypotensive lipids. Am J Ophthalmol. 2002;133(3):393-7. Comment in: Am J Ophthalmol. 2002;133(3):403-5.

10. Wand M, Gaudio AR. Cystoid macular edema associated with ocular hypotensive lipids. Am J Ophthalmol. 2002;133(3):403-5. Comment in: Am J Ophthalmol. 2002;133(3):393-7.

11. Furuichi M, Chiba T, Abe K, Kogure S, Tijima H, Tsukahara S, et al. Cystoid macular edema associated with topical latanoprost in glaucomatous eyes with a normally functioning blood-ocular barrier. J Glaucoma. 2001;10(3):233-6.

12. Irvine SR. A newly defined vitreous syndrome following cataract surgery, interpreted according to recent concepts of the structure of the vitreous. Am J Ophthalmol. 1953;36:599-619.

13. Tranos PG, Wickremasinghe SS, Stangos NT, Topouzis F, Tsinopoulos I, Pavesio CE. Macular edema. Surv Ophthalmol. 2004;49(5):470-90.

14. Antcliff RJ, Stanford MR, Chauhan DS, Graham EM, Spalton DJ, Shilling JS, et al. Comparison between optical coherence tomography and fundus fluorescein angiography for the detection of cystoid macular edema in patients with uveitis. Ophthalmology. 2000;107(3):593-9.

15. Schumer RA, Camras CB, Mandahl AK. Putative side effects of prostaglandin analogs. Surv Ophthalmol. 2002;47(Suppl 1):S219.

16. Yeh PC, Ramanathan S. Latanoprost and clinically significant cystoid macular edema after uneventful phacoemulsification with intraocular lens implantation. J Cataract Refract Surg. 2002;28(10):1814-8. Comment in: J Cataract Refract Surg. 2003;29(6):1053-4; J Cataract Refract Surg. 2003;29(6):1054-5; J Cataract Refract Surg. 2003;29(6):1055-6.

17. Yanoff M, Fine BS, Brucker AJ, Eagle RC Jr. Pathology of human cystoid macular edema. Surv Ophthalmol. 1984;28(Suppl):505-11. 